Supporting Information

\title{
Effects of Cellulose, Hemicellulose, and Lignin on the Structure and Morphology of Porous Carbons
}

Jiang Deng, Tianyi Xiong, Haiyan Wang Anmin Zheng and Yong Wang*

\section{Contents:}

1. Materials ................................................... S1

2. Experiment methods and Characterization .................. S1

3. Figures and schemes ................................. S2-S13

4. Tables .................................................. S14

Materials: $\alpha$-cellulose (Aladdin), $\mathrm{KHCO}_{3}$ (Sinopharm Chemical Reagent Co., Ltd.), Lignin ((TCI Co., Ltd.) were used as received. Xylan from beechwood purchased from Sigma-Aldrich was used as the model polymer of hemicellulose. Water used throughout all experiments was purified through a Millipore system.

Experiment methods:

Synthesis of $\mathrm{C}_{\mathrm{x}-\mathrm{LE}}$ : Firstly, biomass (powder) $(1.2 \mathrm{~g})$ was mixed with $4.8 \mathrm{~g} \mathrm{KHCO}_{3}$. Secondly, the mixture was calcined in a muffle furnace at a programmed temperature under $\mathrm{N}_{2}$ flow (400 $\mathrm{mL} \mathrm{min}^{-1}$ ). The temperature program is as follows: the temperature was raised from ambient temperature to the desired temperature which was maintained for another 1 hour. The heating rate is $15^{\circ} \mathrm{C} \mathrm{min}^{-1}$. Later, it was allowed to cool down naturally. Thirdly, after it cooled down to ambient temperature, a black solid was obtained. Then the black solid was ground into black powder and transferred into a beaker. $200 \mathrm{ml}$ DI water was added to the beaker. The mixture was stirred overnight. Lastly, the solution was filtered and the black solid residue was dried at $70{ }^{\circ} \mathrm{C}$ in an oven overnight.

Synthesis of $\mathrm{C}_{\mathrm{x}}$ : Firstly, the carbon precursor (powder) was calcined in a muffle furnace at the same programmed temperature as $\mathrm{C}_{\mathrm{x}-\mathrm{LE}}$ 's. Then the black solid was ground into black powder.

\section{Characterization}

SEM images were obtained on a Hitachi SU-70. TEM studies were performed on a Hitachi HT-7700 microscope. The diffraction data were collected at room temperature with $2 \theta$ scan range between $10^{\circ}$ and $60^{\circ}$ using a wide-angle X-ray diffraction (Model D/tex-Ultima TV, $1.6 \mathrm{kV}$, Rig-aku, Japan) equipped with $\mathrm{Cu}$ Ka radiation (1.54 $\AA$ ). The Raman spectra were collected on a Raman spectrometer (JY, HR 800) using 514-nm laser. The $\mathrm{N}_{2}$ adsorption-desorption isothermal analysis were determined by 
micromeritics ASAP $2020 \mathrm{HD} 88$, BET equation was used to calculate the surface areas and pore volume and samples were degassed at $200{ }^{\circ} \mathrm{C}$ for $8 \mathrm{~h}$ until the residual pressure was less than $10^{-4} \mathrm{~Pa}$. Thermogravimetric (TG) analysis and TG-MS was performed using METTLER TOLEDO TGA/DSC 1100SF. All ${ }^{13} \mathrm{C}$ NMR experiments were carried out on a Varian Infinityplus-300 spectrometer with the use of a $4 \mathrm{~mm}$ double-resonance MAS probe at a spinning rate of $10 \mathrm{kHz}$. The Larmor resonance frequencies for $1 \mathrm{H}$ and ${ }^{13} \mathrm{C}$ were 299.78 and $75.38 \mathrm{MHz}$, respectively. ${ }^{13} \mathrm{C}$ MAS NMR spectra with high power proton decoupling were recorded using a $\pi / 2$ pulse length of $4.1 \mathrm{us}$ and a recycle delay of $10 \mathrm{~s}$. The chemical shift of ${ }^{13} \mathrm{C}$ was externally referenced to adamantane.

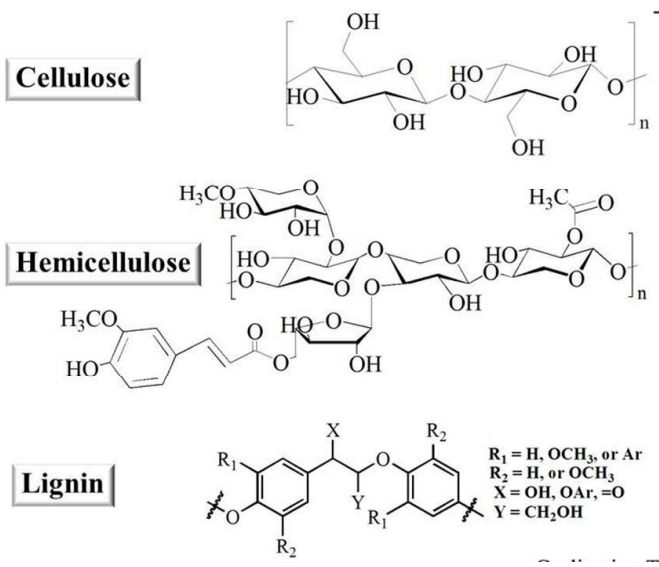

Scheme S1. the possible structure revolution of cellulose and lignin under the thermal treatment with assistance of $\mathrm{KHCO}_{3}{ }^{1}$ 

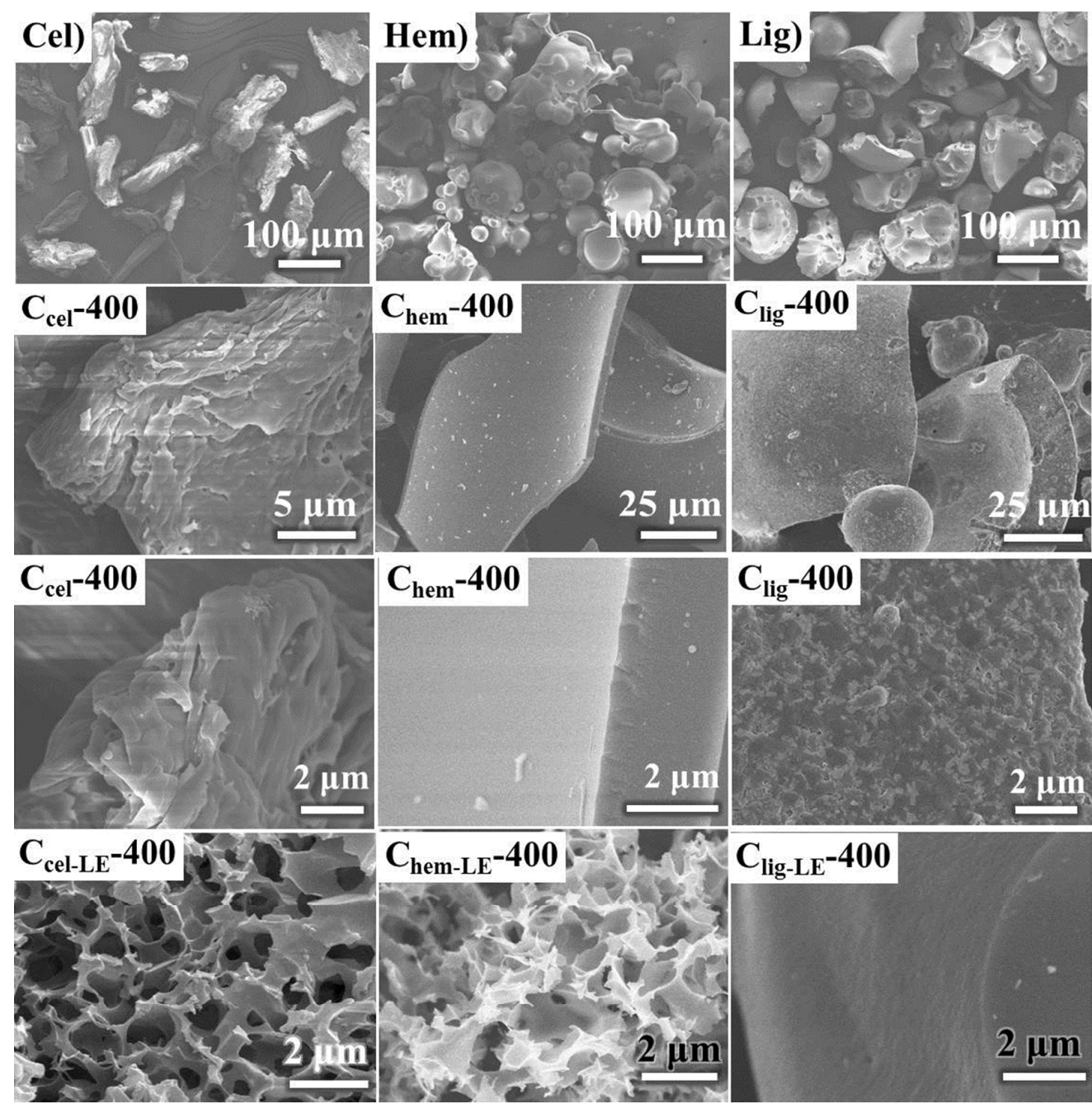

Figure S1. Morphologies evolution of cellulose, hemicellulose and lignin

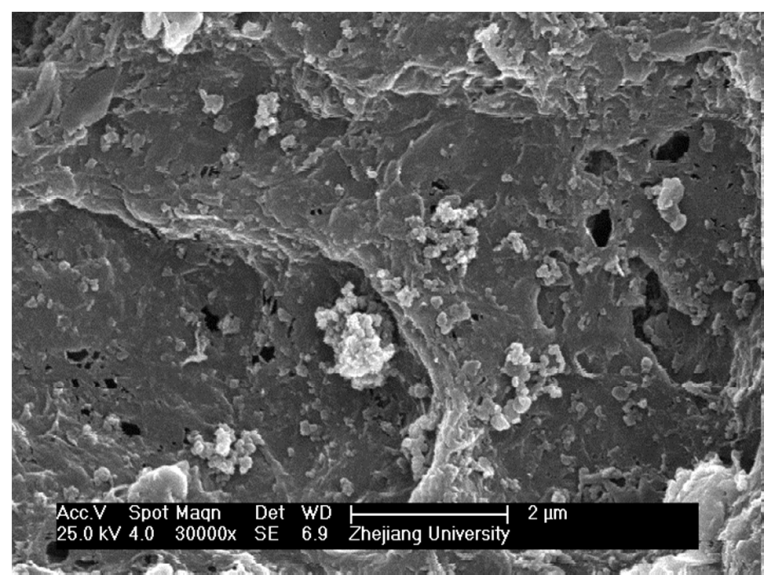

Figure S2. SEM image of $\mathrm{C}_{\text {lig-LE }}-900$ in absence of ultrasonic-assisted dispersion 

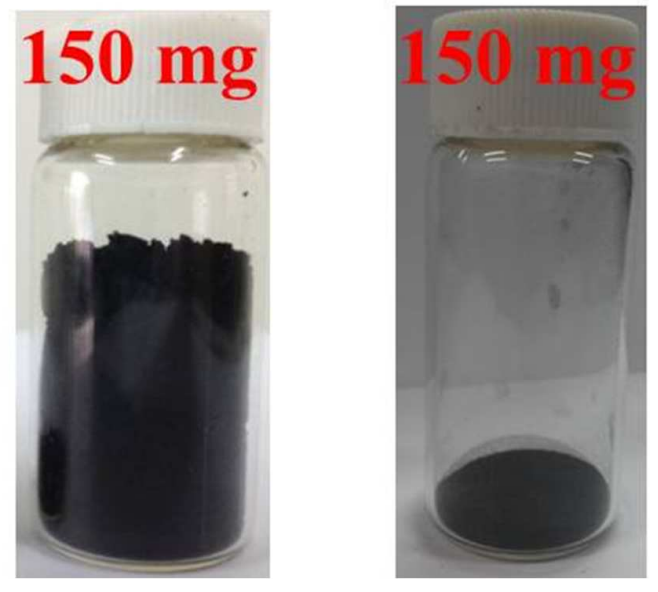

Figure S3. Digital photos of $\mathrm{C}_{\text {cel-LE }}\left(\right.$ left) and $\mathrm{C}_{\text {lig-LE }}$ (right)
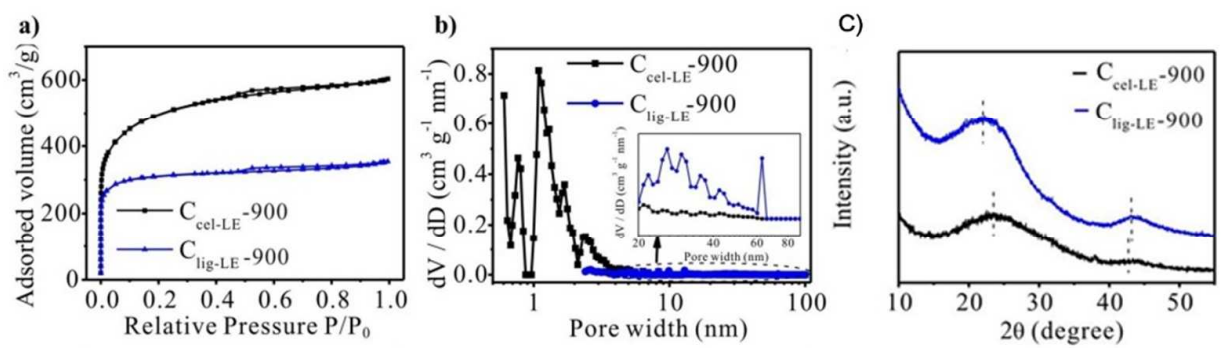

Figure S4. (a) $\mathrm{N}_{2}$ isotherms and (b) PSDs using the DFT model of the $\mathrm{C}_{\text {cel-LE}}-900$ and $\mathrm{C}_{\text {lig-LE }}-900$, (c) XRD results of $\mathrm{C}_{\text {cel-LE }}-900$ and $\mathrm{C}_{\text {lig-LE }}-900$

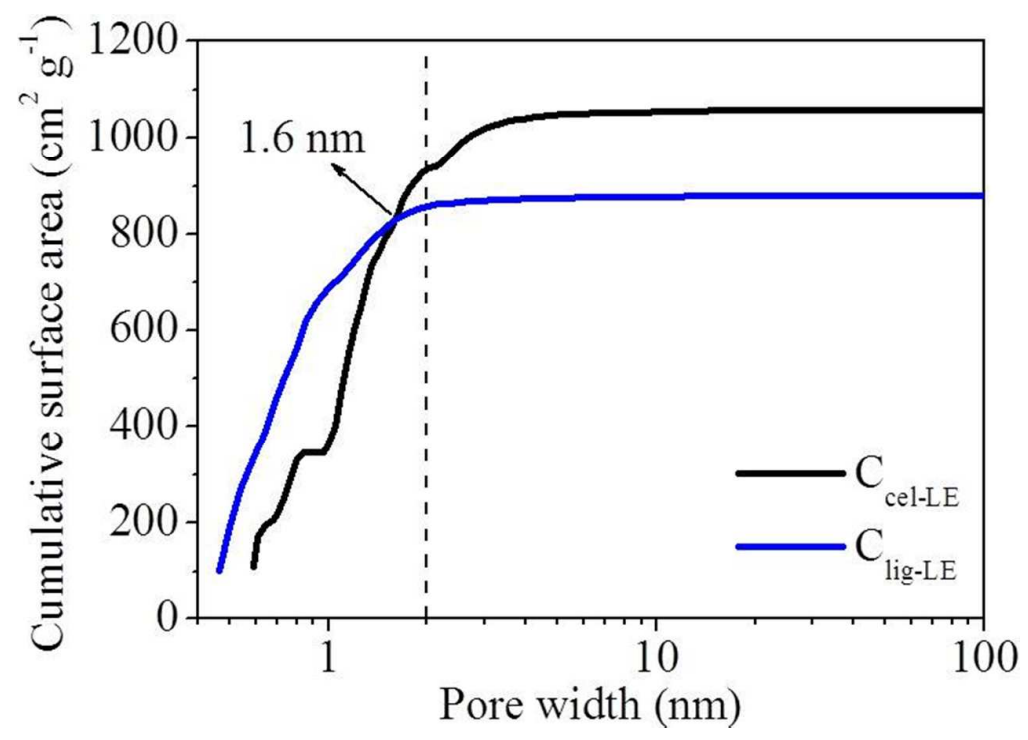

Figure S5. Cumulative surface area of $\mathrm{C}_{\text {cel-LE }}$ and $\mathrm{C}_{\text {lig-LE }}$ 

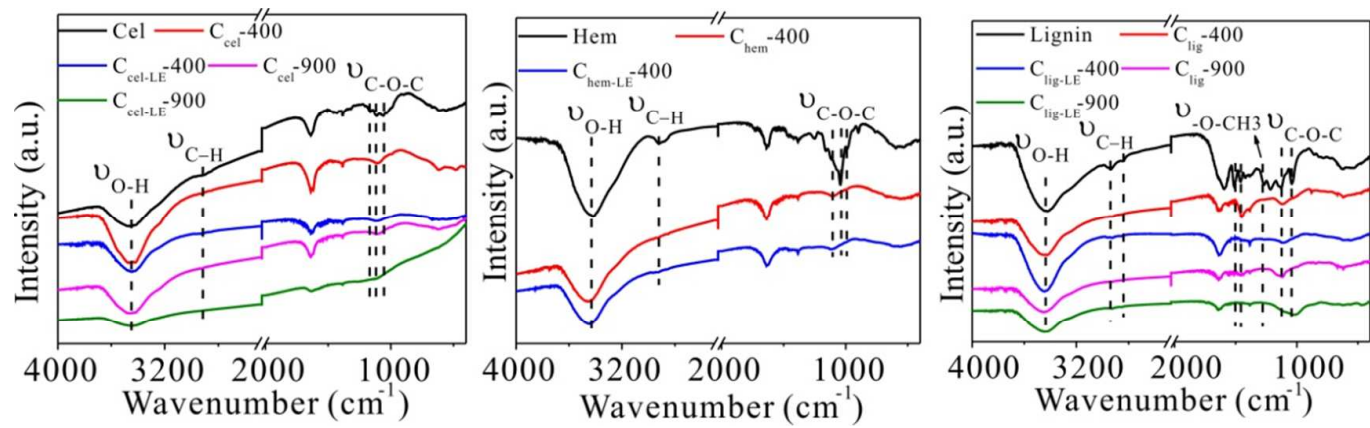

Figure S6. FTIR spectroscopy results of cellulose, hemicellulose, lignin and their derived pyrolytic carbon.

According to the FTIR spectroscopy of cellulose and its derived carbon, the intensity of $v_{\mathrm{O}-\mathrm{H}}, v_{\mathrm{C}-\mathrm{H}}$ and $v_{\mathrm{C}-\mathrm{O}-\mathrm{C}}$ decrease with the increase of the calcination termperature. The same tendency also happen to hemicellulose and its derived carbon as well as lignin and its correspondingly derived products. Obviously, we can still see the peaks of aromatic skeletal vibrations and asymmetric $\mathrm{C}-\mathrm{H}$ bending from methoxyl group in $\mathrm{C}_{\text {lig }}-400$, which suggest the robust aromatic structure is to some extent stable. The absorbtion peak at $\sim 1643 \mathrm{~cm}^{-1}$ observed in amost all the samples attribute to the adsorbed water. Comparing the IR results of $\mathrm{C}_{\text {cel- }}-900$ and $\mathrm{C}_{\text {cel-LE }}-900$, the weaker $v_{\mathrm{O}-\mathrm{H}}$ in $\mathrm{C}_{\text {cel-LE}}-900$ indicated activator contributed to the decomposation of $-\mathrm{OH}$ leading to less hydroxyl. ${ }^{2}$

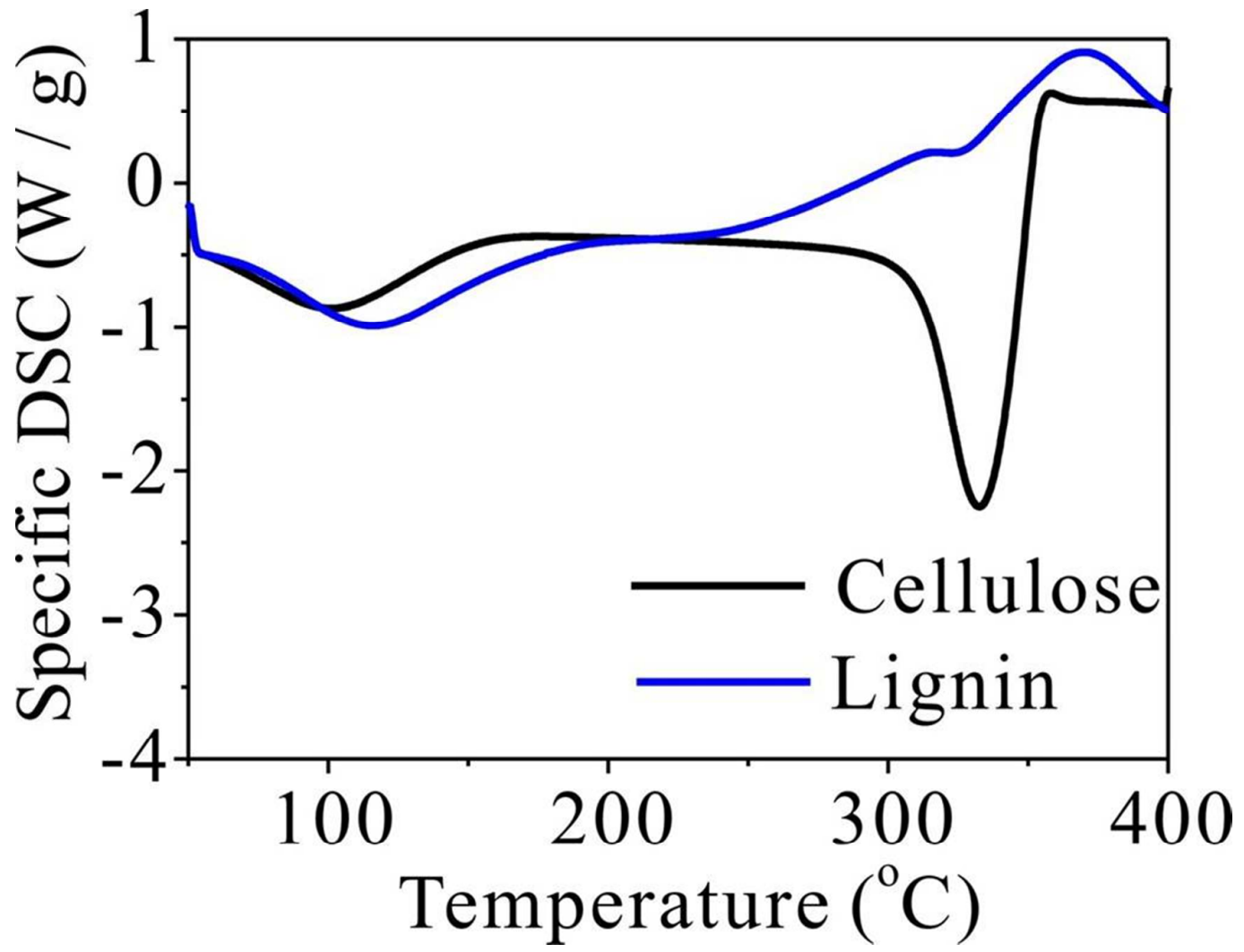

Figure S7. DSC measurements of cellulose and lignin 


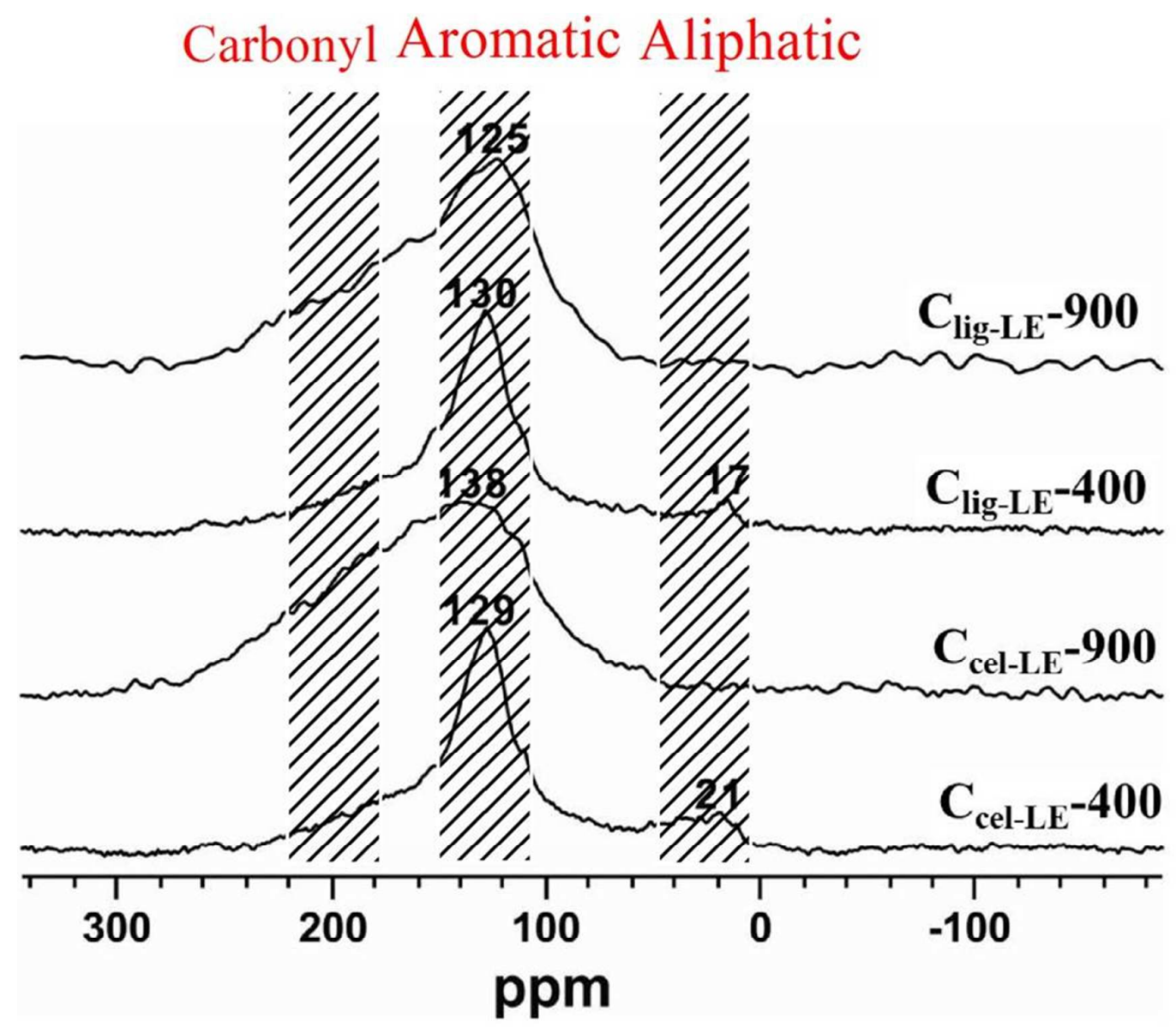

Figure S8. Solid-state NMR results of $\mathrm{C}_{\text {lig-LE }}-900, \mathrm{C}_{\text {lig-LE }}-400, \mathrm{C}_{\text {cel-LE }}-900$, and $\mathrm{C}_{\text {cel-LE }}-400$

$\mathrm{C}_{\text {lig-LE- }} 900\left(\mathrm{C}_{\text {cel-LE}}-900\right)$ represents the final thermal treated temperature is $900{ }^{\circ} \mathrm{C}$, while is $400{ }^{\circ} \mathrm{C}$ for $\mathrm{C}_{\text {lig-LE }}-400\left(\mathrm{C}_{\text {cel-LE}}-400\right)$. The peaks of $\mathrm{C}_{\text {cel-LE}}-400$ at $21 \mathrm{ppm}$ corresponding to the existence of $-\mathrm{CH}_{2}$ - which turn to disappear when the temperature is set at $900{ }^{\circ} \mathrm{C}$. Furthermore, the main peak of $\mathrm{C}_{\text {cel-LE }}-900$ is broader than that of $\mathrm{C}_{\text {cel-LE}}-400$ whose location also moves from $129 \mathrm{ppm}$ to $138 \mathrm{ppm}$. Those evolutions suggest that the aromatization degree improve with the increasing of temperature. Lots of adjacent hydroxyl in cellulose undergo dehydration and formed $\mathrm{C}=\mathrm{O}$ leading to a red shift of the main peak. However, the main peak of $\mathrm{C}_{\text {lig-LE }}-900$ own higher shift comparing with $\mathrm{C}_{\text {lig-LE}}-400$, which indicate that lignin favor to form aromatic structure with less oxygen-containing function groups. ${ }^{3}$ 

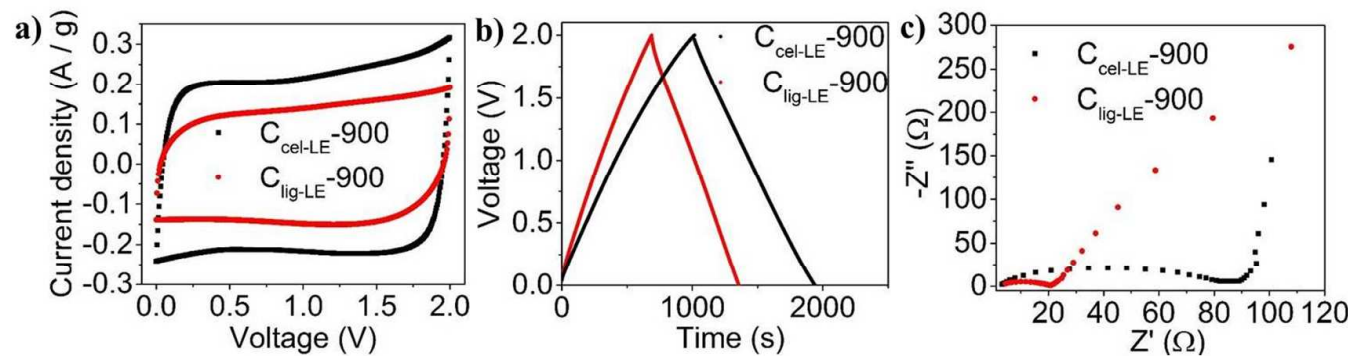

Figure S9. The $\mathrm{CV}$ curves of $\mathrm{C}_{\text {cel-LE }}-900$ and $\mathrm{C}_{\text {lig-L }} \mathrm{E}-900$ tested at $5 \mathrm{mv} / \mathrm{s}$, (b) Galvanostatic charge/discharge curves of $\mathrm{C}_{\text {cel-LE }}-900$ and $\mathrm{C}_{\text {lig-LE }}-900$ tested at $0.1 \mathrm{~A} / \mathrm{g}$; (c) Nyquist plots of $\mathrm{C}_{\text {cel-LE}}-900$ and $\mathrm{C}_{\text {lig-LE }}-900$ in the frequency range of $100 \mathrm{kHz}$ to $10 \mathrm{mHz}$ in $1 \mathrm{M} \mathrm{Bmin} \mathrm{BF}_{4} / \mathrm{AN}$ electrolyte.

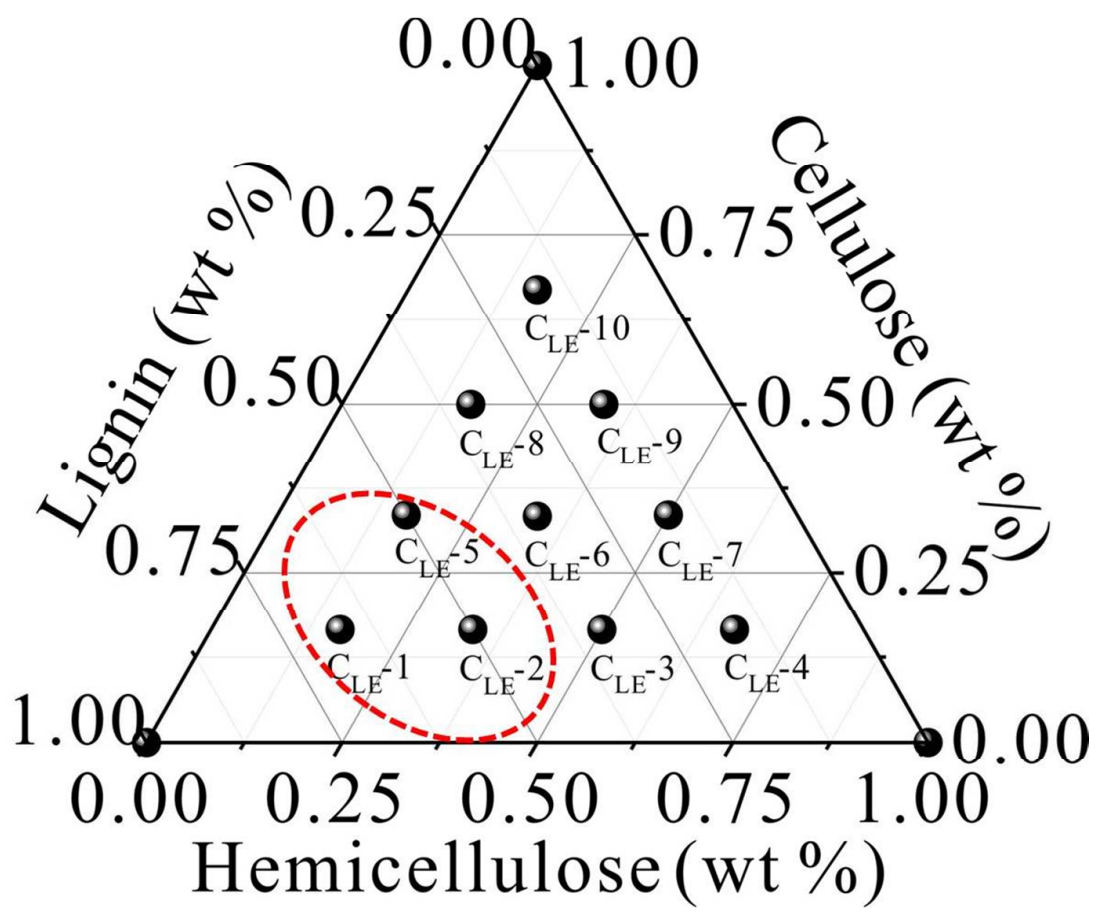

Figure S10. Ternary plot of the carbonaceous materials derived from leavening method with different ratio of cellulose, lignin, and hemicellulose. (refer to Table S2 for the formulation of each sample) 
On the basis of the SEM analysis (Figure 11-1 and 11-2), the products circled by red line in Figure S11 give inhomogeneous morphologies, which contain both macropores and bulk monoliths. Although the macroposity can be obtained by introducing cellulose into the raw materials, higher content of lignin $(>50 \%)$ would still result in partial morphologies without macroposity.

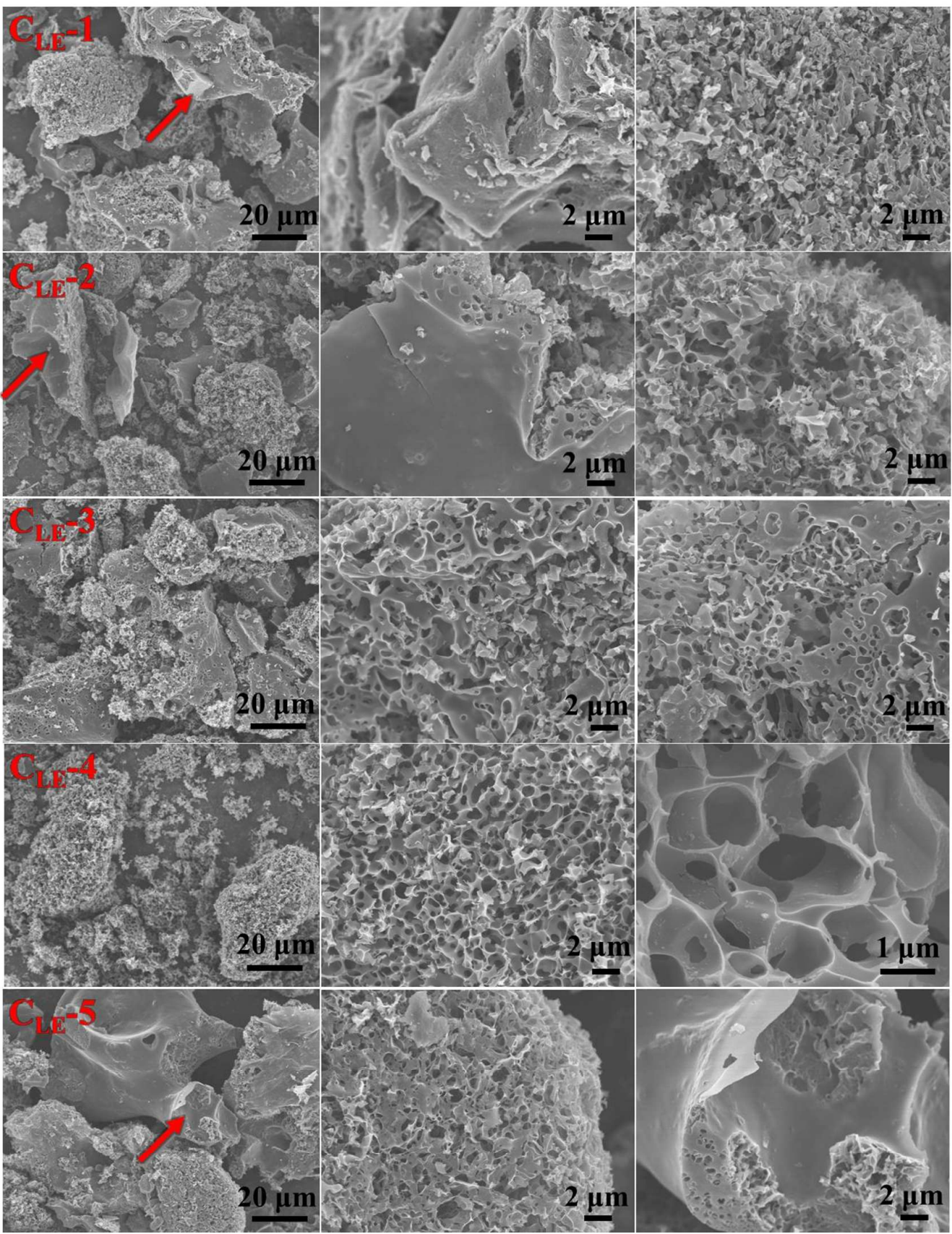

Figure S11-1. SEM images of $\mathrm{C}_{\mathrm{LE}^{-}} 1, \mathrm{C}_{\mathrm{LE}^{-}}-2, \mathrm{C}_{\mathrm{LE}^{-}} 3, \mathrm{C}_{\mathrm{LE}^{-}}$, and $\mathrm{C}_{\mathrm{LE}^{-}} 5$ 


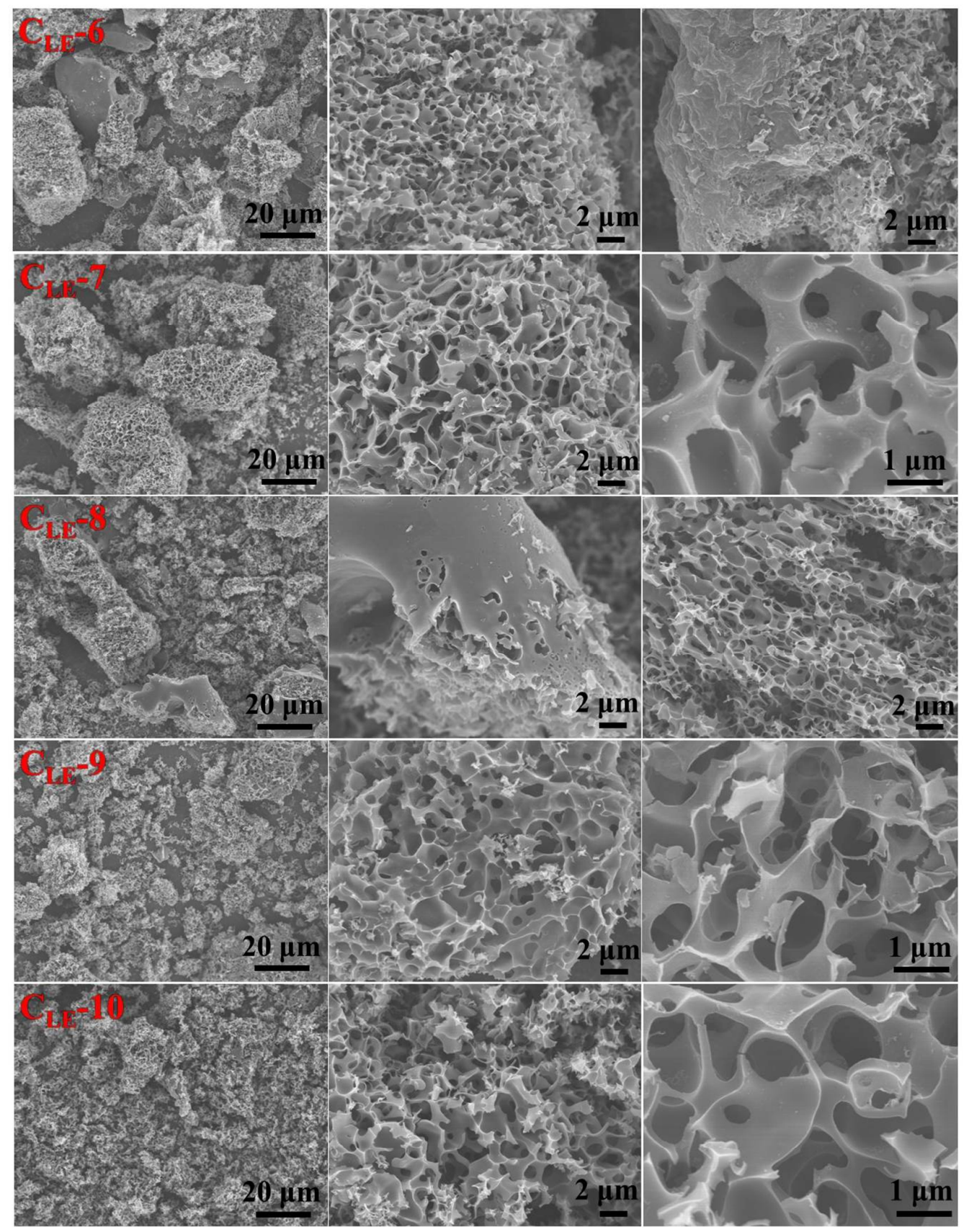

Figure S11-2. SEM images of $\mathrm{C}_{\mathrm{LE}^{-}}-6, \mathrm{C}_{\mathrm{LE}^{-}}-7, \mathrm{C}_{\mathrm{LE}^{-}}-8, \mathrm{C}_{\mathrm{LE}^{-}}-9$, and $\mathrm{C}_{\mathrm{LE}^{-}}-10$

All the SEM images show a macroporous structure. However, partial bulky morphologies can still be seen for $\mathrm{C}_{\mathrm{LE}}-1, \mathrm{C}_{\mathrm{LE}}-2$, and $\mathrm{C}_{\mathrm{LE}}-5$ labeled by the red arrow. The $\mathrm{N}_{2}$ sorption analyses (Figure S12-1 and S12-2) indicate that all the products contain macro-, meso-, and micropores giving a hierarchically porous structure. 

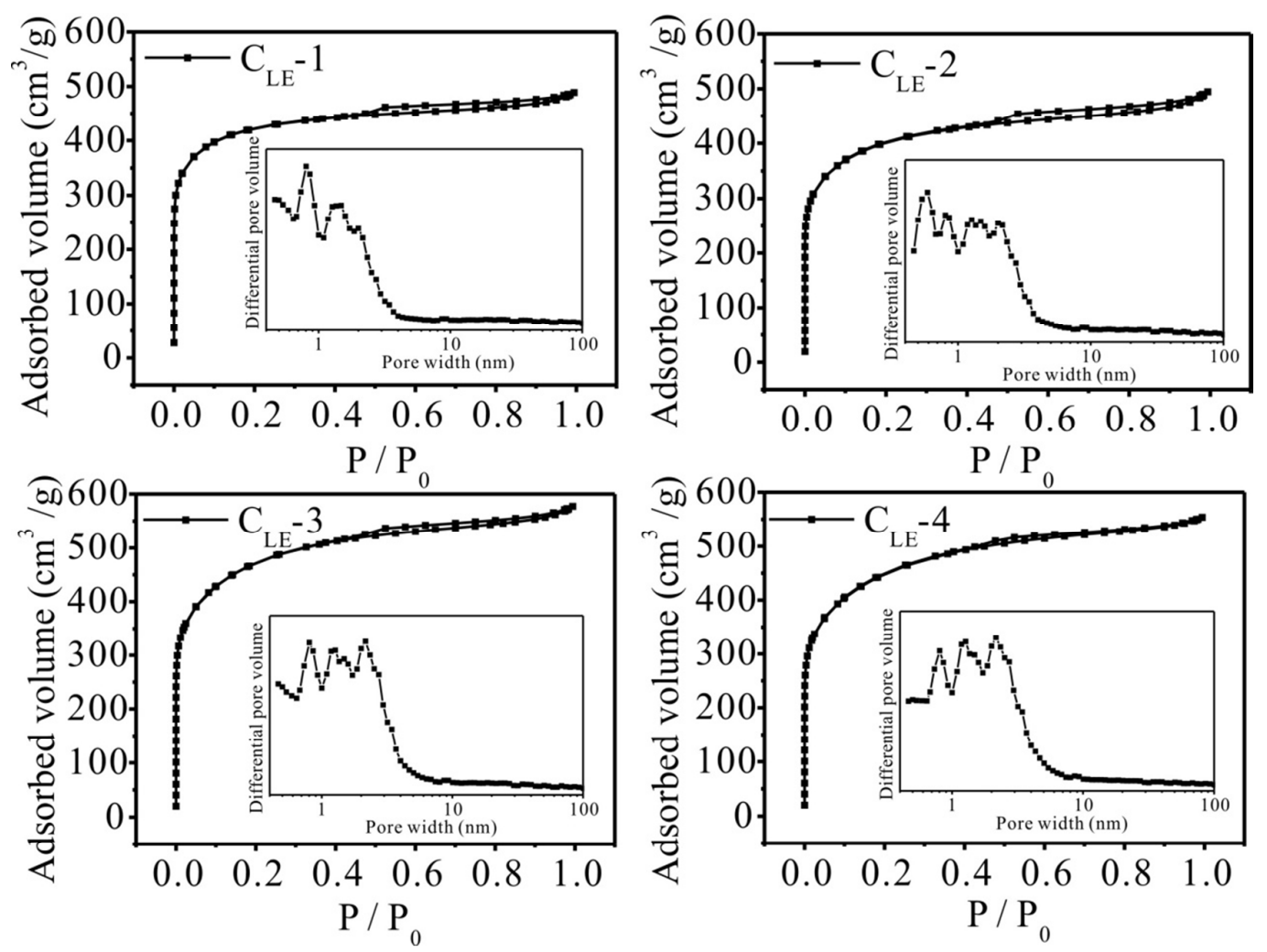

Figure S12-1. $\mathrm{N}_{2}$ sorption isotherms and PSD curves of $\mathrm{C}_{\mathrm{LE}^{-}}-\mathrm{C}_{\mathrm{LE}^{-}}-2, \mathrm{C}_{\mathrm{LE}}-3$, and $\mathrm{C}_{\mathrm{LE}}-4$ 

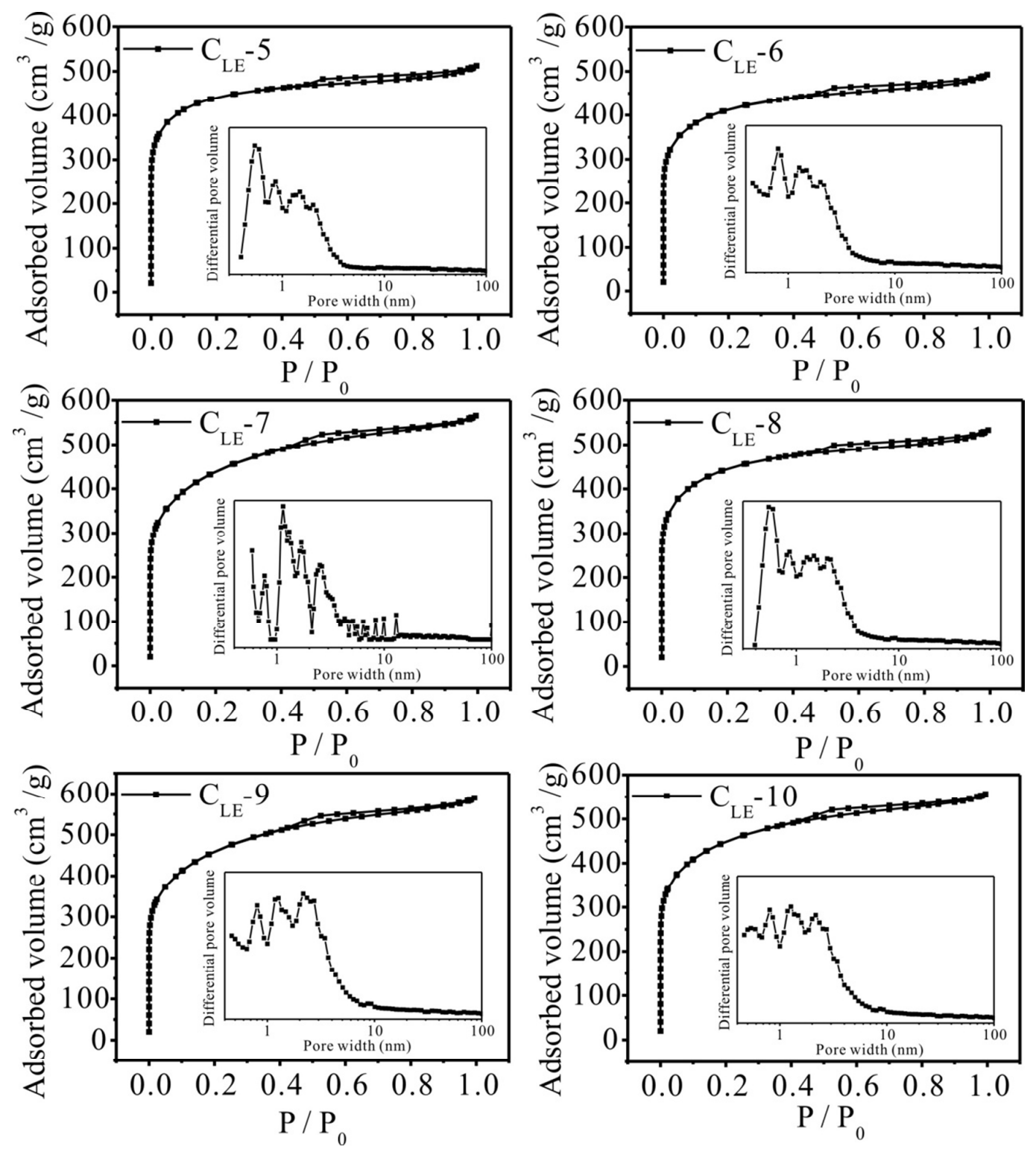

Figure S12-2. $\mathrm{N}_{2}$ sorption isotherms and PSD curves of $\mathrm{C}_{\mathrm{LE}}-5, \mathrm{C}_{\mathrm{LE}}-6, \mathrm{C}_{\mathrm{LE}}-7, \mathrm{C}_{\mathrm{LE}}-8$, $\mathrm{C}_{\mathrm{LE}}-9$, and $\mathrm{C}_{\mathrm{LE}^{-}}-10$ 

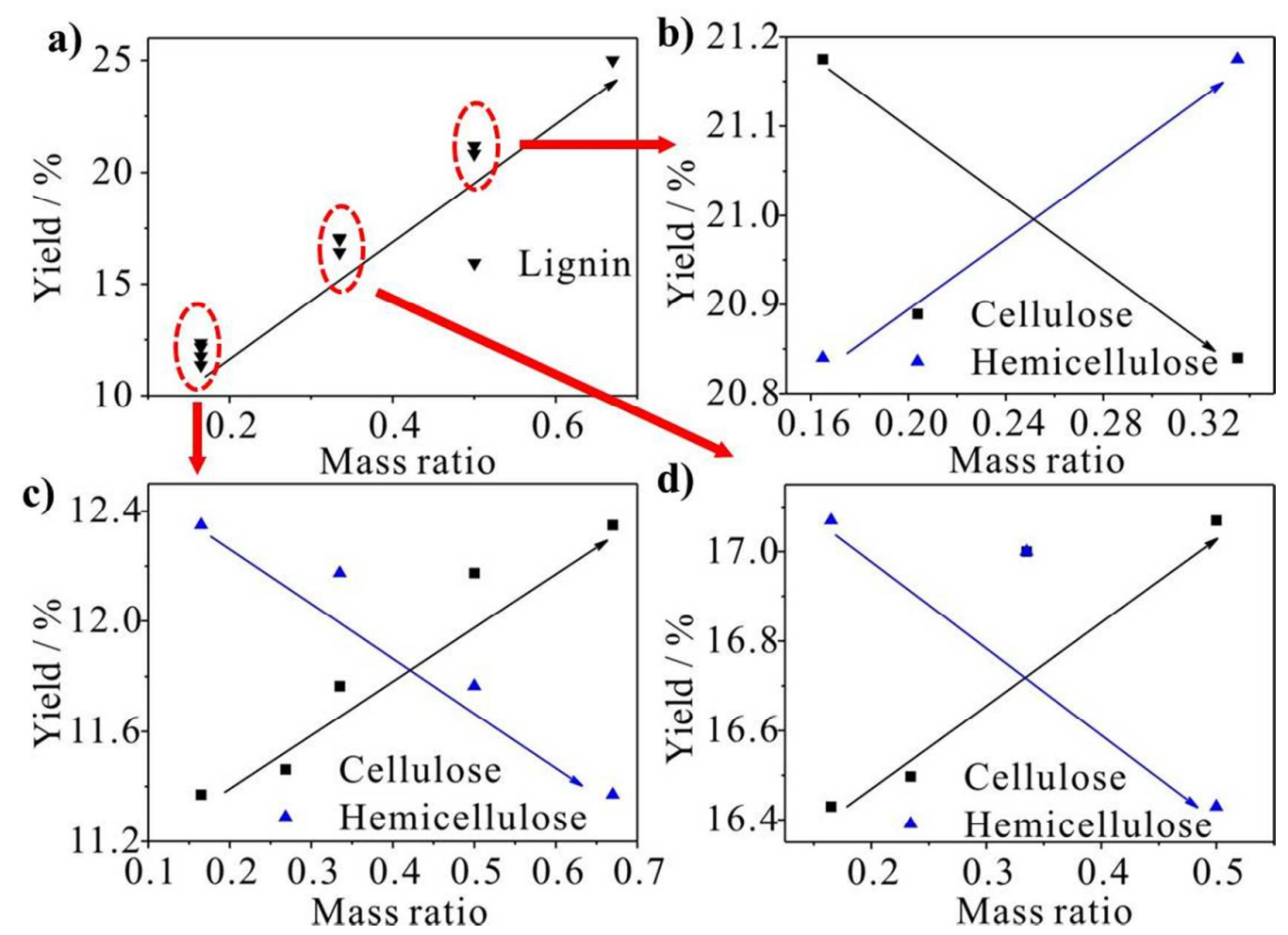

Figure S13. the yield of products origin from the tertiary mixture via leavening method with different mass ratio of each part

Figure S13a show that the total yields highly depend on the mass ration of lignin, which indicate that the aromatic rings in lignin tend to undergo condensation process and produce carbonaceous materials. Figure S13c and S13d show that the mass ratios of cellulose make a big influence on the yield ruling out the impression of lignin. However, this affection reverses when the mass ratio of lignin is $50 \%$. (Figure S13b) It seems a proper mass ratio of each component is also important for a high yield. Also, each component (cellulose, lignin, and hemicellulose) would contribute to the products of the mixture through leaven method.

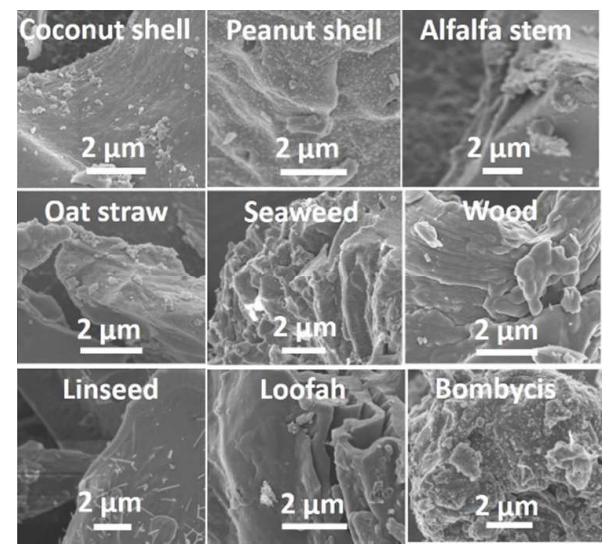

Figure S14 SEM images of carbon materials derived from direct pyrolysis on the basis of different kinds of crude biomass 
Figure S14 shows that products derived from direct pyrolysis on the basis of different kinds of crude biomass exhibit bulky morphology.

Table S1. The parameters of specific surface area, pore volume and average pore width calculated from nitrogen adsorption measurements.

\begin{tabular}{|c|c|c|c|c|c|c|}
\hline \multirow{2}{*}{ Sample } & \multirow{2}{*}{ Yield (\%) } & \multirow{2}{*}{$\begin{array}{c}\mathbf{S}_{\mathrm{BET}}{ }^{\mathrm{a}} \\
{\left[\mathbf{m}^{2} \mathbf{g}^{-1}\right]}\end{array}$} & \multicolumn{3}{|c|}{ Pore volume $\left(\mathrm{cm}^{3} \mathrm{~g}^{-1}\right)$} & \multirow{2}{*}{$\begin{array}{l}\mathbf{V}_{\text {Micro }} / \\
\mathbf{V}_{\text {Total }}\end{array}$} \\
\hline & & & $\mathbf{V}_{\text {Total }}^{\mathbf{b}}$ & $\mathbf{V}_{\text {Micro }}{ }^{c}$ & $\mathbf{V}_{\text {Macro\&Meso }}$ & \\
\hline$C_{\text {cel-LE }}-900$ & 8.7 & 1635 & 0.93 & 0.74 & 0.19 & $80 \%$ \\
\hline$C_{\text {hem-LE }}-900$ & $\sim 0$ & - & - & - & - & - \\
\hline$C_{\text {lig-LE }}-900$ & 39 & 976 & 0.54 & 0.47 & 0.07 & $87 \%$ \\
\hline $\mathrm{C}_{\mathrm{cel}^{-}}-900$ & 16 & 527 & 0.28 & 0.26 & 0.02 & $93 \%$ \\
\hline$C_{\text {hem }}-900$ & $\sim 0$ & - & - & - & - & - \\
\hline$C_{\text {lig }}-900$ & 51 & 73 & 0.042 & 0.030 & 0.012 & $71 \%$ \\
\hline$C_{\text {cel-LE }}-400$ & 16 & 124 & 0.09 & 0.045 & 0.045 & $50 \%$ \\
\hline$C_{\text {hem LE }}-400$ & 7 & 197 & 0.13 & 0.08 & 0.05 & $62 \%$ \\
\hline $\mathrm{C}_{\text {lig-LE }}-400$ & 51 & - & - & - & - & - \\
\hline $\mathrm{C}_{\mathrm{cel}^{-}-400}$ & 22 & 0.59 & - & - & - & - \\
\hline$C_{\text {hem }}-400$ & 23 & 11 & 0.0049 & 0.0065 & - & - \\
\hline $\mathrm{C}_{\text {lig }}-400$ & 68 & - & - & - & - & - \\
\hline
\end{tabular}

${ }^{\mathrm{a}}$ Brunuaer-Emmett-Teller surface area. ${ }^{\mathrm{b}}$ Calculated by single-point adsorption at relative pressure of 0.98 .

${ }^{c}$ Calculated by V-t plot method. ${ }^{d}$ Calculated by BJH method from the desorption isotherm linear plot.

Table S2 the yield and specific surface area of products origin from the tertiary mixture via leavening method with different mass ratio of each part.

\begin{tabular}{|c|c|c|c|c|c|c|c|c|c|c|}
\hline & $\mathrm{LE}^{-1}$ & $\mathrm{C}_{\mathrm{LE}}-2$ & $\mathrm{C}_{\mathrm{LE}}-3$ & $\mathrm{C}_{\mathrm{LE}^{-4}}$ & $\mathrm{C}_{\mathrm{LE}}-5$ & $\mathrm{C}_{\mathrm{LE}^{-6}}$ & $\mathrm{C}_{\mathrm{LE}^{-7}}$ & $\mathrm{C}_{\mathrm{LE}}-8$ & $\mathrm{C}_{\mathrm{LE}^{-}}-9$ & $\mathrm{C}_{\mathrm{LE}_{\mathrm{E}}}-10$ \\
\hline Cellu & 032 & 033 & 0.33 & 0.33 & 0.67 & 0.67 & 0.67 & 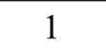 & 1 & 133 \\
\hline 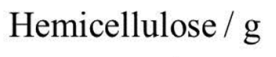 & & 7 & 1 & 4 & 0.33 & 0.67 & 1 & 0.33 & 0.67 & 0.3 \\
\hline & 1 & 1 & 0.67 & 33 & 1 & 0.67 & 0.33 & 0.67 & 0.33 & 0.33 \\
\hline $\mathrm{M}^{*} / \mathrm{g}$ & 0.50 & 42 & 0.33 & 0.23 & 0.42 & 0.46 & 0.28 & 0.35 & 0.25 & 0.23 \\
\hline 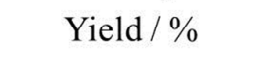 & 25 & 21 & 16 & 11 & 21 & 34 & 24 & 34 & 24 & 25 \\
\hline $\mathrm{S}_{\mathrm{BET}}{ }^{* *} / \mathrm{cm}^{2} \mathrm{~g}^{-1}$ & 1350 & 1307 & 1551 & 1487 & 1409 & 1335 & 1464 & 1445 & 1533 & 1476 \\
\hline
\end{tabular}

${ }^{*} \mathrm{M}$ represents the mass of final product.

${ }^{* *} \mathrm{~S}_{\mathrm{BET}}$ is the specific surface area calculated by BET model.

1. Braun, J.; Holtman, K.; Kadla, J., Lignin-based carbon fibers: Oxidative thermostabilization of kraft lignin. Carbon 2005, 43 (2), 385-394.

2. Schwanninger, M.; Rodrigues, J. C.; Pereira, H.; Hinterstoisser, B., Effects of 
short-time vibratory ball milling on the shape of FT-IR spectra of wood and cellulose. Vibrational Spectroscopy 2004, 36 (1), 23-40.

3. Baccile, N.; Falco, C.; Titirici, M.-M., Characterization of biomass and its derived char using 13C-solid state nuclear magnetic resonance. Green Chem. 2014, 16 (12), 4839-4869. 